\title{
PRODUCTS OF SEQUENTIAL SPACES
}

\author{
YOSHIO TANAKA
}

Dedicated to Professor Kiiti Morita on the occasion of his 60 th birthday

\begin{abstract}
S. P. Franklin introduced the notion of a sequential space and characterized such spaces as being precisely the quotient images of metric spaces.

In this paper we investigate a necessary and sufficient condition for the product of a first countable space with a sequential space to be sequential, and we consider the property "sequential space" in $X^{\omega}$.
\end{abstract}

1. Introduction. Throughout this paper, by a space we shall mean a regular, $T_{1}$-space.

The symbol $N$ will refer to the set of natural numbers.

Let us recall that a space $X$ is sequential [2], if a subset $F$ of $X$ is closed whenever $F \cap C$ is closed in $C$ for every convergent sequence $C$ together with its limit point.

Metric spaces, or more generally Fréchet (= Fréchet-Urysohn) spaces are sequential. Sequential spaces are $k$-spaces.

As is well known [2], the product of a sequential space with a separable metric space need not be sequential.

As for the product of a sequential space with a first countable space, our main theorem, which will be established in $\S 3$, reads as follows:

TheOREM 1.1. Let $X$ be a Fréchet space, or a sequential space each of whose points is a $G_{\delta^{-s e t}}$ (or equivalently, a $k$-space each of whose points is a $G_{\delta^{-s e t}}$ ). Let $Y$ be first countable. Then $X \times Y$ is sequential if and only if $X$ is strongly Fréchet, or $Y$ is locally countably compact.

In the necessity, the property "each point of $X$ is a $G_{\delta^{-}}$-set" is essential.

According to Siwiec [12], a space $X$ is called strongly Fréchet (= countably bisequential in the sense of E. Michael [6]) if, whenever $\left\{F_{n} ; n \in N\right\}$ (or simply $\left.\left\{F_{n}\right\}\right)$ is a decreasing sequence accumulating at $x$ in $X$, there exist $x_{n} \in F_{n}$ such that the sequence $\left\{x_{n} ; n \in N\right\}$ (or simply $\left\{x_{n}\right\}$ ) converges to $x$.

Metric spaces are strongly Fréchet. Strongly Fréchet spaces are Fréchet.

As a special class of sequential spaces, we shall consider symmetrizable spaces.

According to A. V. Arhangel'skii [1], a space $X$ is symmetrizable, if there is a real valued, nonnegative function $d$ defined on $X \times X$ satisfying the

Received by the editors November 3, 1974.

AMS (MOS) subject classifications (1970). Primary 54B10.

Key words and phrases. Sequential spaces, Fréchet spaces, strongly Fréchet spaces, symmetrizable spaces, semimetrizable spaces. 
following: (1) $d(x, y)=0$ iff ( = if and only if) $x=y,(2) d(x, y)=d(y, x)$, and (3) $A \subset X$ is closed iff $d(x, A)>0$ for any $x \in X-A$.

If we replace (3) by (3)': $x \in \bar{A}$ iff $d(x, A)=0$, then such a space $X$ is called semimetrizable [4].

Metric spaces, or more generally semimetrizable spaces, are symmetrizable.

It has been shown [14] that the product of a countable, symmetrizable space with a separable metric space need not be symmetrizable.

In the following theorem, we establish a necessary and sufficient condition for the product of a symmetrizable space with a semimetrizable space to be symmetrizable.

THEOREM 1.2. Let a symmetrizable space $X$ be paracompact, or more generally meta-Lindelöf (i.e. every open covering has a point-countable open refinement), or have each point a $G_{\delta^{-}}$set. Let $Y$ be semimetrizable. Then $X \times Y$ is symmetrizable if and only if $X$ is semimetrizable, or $Y$ is locally compact.

As for the property "sequential space" in the product $X^{\omega}$ of countably many copies of $X$, in $\S 4$, we will have

THEOREM 1.3. Let $X$ have one of the three properties listed below:

(i) $\boldsymbol{\aleph}_{0}$-space in the sense of E. Michael [5],

(ii) closed image of a metric space,

(iii) $C W$-complex in the sense of Whitehead.

If $X^{\omega}$ is sequential, then $X$ is metrizable.

It follows from [14] that there is a sequential space (in fact, a symmetrizable space) $X$ such that $X^{2}$ is not sequential.

In this respect, it will be shown that the higher power $X^{\omega}$ can also behave unpredictably.

That is, there is a space $X$ such that $X^{n}$ is sequential (in fact, symmetrizable) for all $n \in N$, but $X^{\omega}$ is not even sequential.

2. Preliminaries. As a weaker condition than " $X$ is strongly Fréchet", we shall of ten make use of the following condition (C) on $X$.

(C) Let $\left\{F_{n}\right\}$ be a decreasing sequence accumulating at $x \in X$. Then there exist $x_{n} \in F_{n}$ such that the sequence $\left\{x_{n}\right\}$ converges to some point $x^{\prime} \in X$.

LEMMA 2.1. (A) Let $X$ be a Fréchet space, or a space each of whose points is $a G_{\delta^{-}}$set. If $X$ satisfies condition $(\mathrm{C})$, then $X$ is strongly Fréchet.

(B) Let $X$ be a symmetrizable space satisfying condition (C). If $X$ is also metaLindelöf, or each point of $X$ is a $G_{\delta^{-}}$set. Then $X$ is semimetrizable.

Proof. (A) In case $X$ is Fréchet, in view of the proof of [11, Theorem 5.1], $X$ is strongly Fréchet.

In case each point of $X$ is a $G_{\delta^{-}}$set, it may be proved directly that $X$ is strongly Fréchet.

(B) We shall prove that $X$ is Fréchet. Because of part (A), we need only consider the case where $X$ is meta-Lindelöf. Let $D$ be a countable subset of $X$. Then the meta-Lindelöf space $\bar{D}$ is separable, hence is Lindelöf. Since $\bar{D}$ is symmetrizable, by [10, Theorem 2] $\bar{D}$ is hereditarily Lindelöf, and hence each point of $\bar{D}$ is a $G_{\delta^{-}}$-set in $\bar{D}$. Since $\bar{D}$ satisfies condition (C), by part (A), $\bar{D}$ is strongly Fréchet. Then $D$ is Fréchet. Thus each countable subset of $X$ is 
Fréchet. Hence $X$ is Fréchet by [6, Proposition 8.7]. Thus $X$ is first countable, for Fréchet, symmetrizable spaces are first countable [1]. Since first countable, symmetrizable spaces are semimetrizable, $X$ is semimetrizable.

LemMa 2.2. Let $X$ be sequential. If $X$ does not satisfy condition (C), then there is a countable, metric space $Y_{0}$ such that $X \times Y_{0}$ is not sequential.

Proof. Since $X$ does not satisfy condition (C), there is a point $x_{0}$ of $X$, and a decreasing sequence $\left\{A_{n}\right\}$ accumulating at $x_{0}$ satisfying

(K) If $x_{n} \in A_{n}$, then the sequence $\left\{x_{n}\right\}$ has no limits. Since $X$ is sequential and $x_{0} \in \bar{A}_{n}$ for $n \in N$, by [6, Lemma 8.3 and Proposition 8.5], there is a sequence $\left\{C_{n}\right\}$ of countable subsets of $X$ such that $C_{n} \subset A_{n}$ and $x_{0} \in \overline{C_{n}}$.

Let $Y_{0}=\cup_{n=1}^{\infty}\left\{C_{n} \times\{n\}\right\} \cup\left\{x_{0}\right\}$, and topologize $Y_{0}$ as follows:

Let each point of $\cup_{n=1}^{\infty} C_{n} \times\{n\}$ be open, and $\left\{V_{n}\left(x_{0}\right)\right\}$ be a countable local base at $x_{0}$, where $V_{n}\left(x_{0}\right)=\cup_{i \geqq n}^{\infty}\left\{C_{i} \times\{i\}\right\} \cup\left\{x_{0}\right\}$. Then $Y_{0}$ is a metric space, which is not locally compact. Let $A=\left\{(x,(x, n)) \in X \times Y_{0} ; n \in N, x \in C_{n}\right\}$. Then $\left(x_{0}, x_{0}\right) \in \bar{A}-A$. Thus $A$ is not closed in $X \times Y_{0}$.

Suppose that $X \times Y_{0}$ is sequential. Then a subset $F$ of $X \times Y_{0}$ is closed whenever $F \cap(C \times K)$ is closed in $C \times K$ for every convergent sequence $C$ in $X$ and every convergent sequence $K$ in $Y_{0}$. Let $C, K$ be convergent sequences in $X, Y_{0}$ respectively, and let $B=A \cap(C \times K)$. To see that $B$ is a closed subset of $C \times K$, let $z \in C \times K-B$. We need only consider the case $z=\left(x, x_{0}\right)$. The condition $(\mathrm{K})$ implies that there is $A_{i_{0}}$ which contains no elements of $C$. Then there is a neighborhood $X \times V_{i_{0}}\left(x_{0}\right)$ of $z$ which is disjoint from the set $B$. Thus $B$ is closed in $C \times K$. Hence $A$ is closed in $X \times Y_{0}$, which is a contradiction. Therefore $X \times Y_{0}$ is not sequential.

LEMMA 2.3. Let $X$ be first countable. If $X$ is not locally countably compact, then the space $Y_{0}$ in Lemma 2.2 is a closed subset of $X$.

Proof. By the hypotheses for $X$, there is a point $x_{0}$ of $X$, and a countable local base $\left\{U_{n}\right\}$ at $x_{0}$ such that each $\bar{U}_{n}$ is not countably compact.

By induction, we can obtain a sequence $\left\{C_{n_{k}}\right\}$ of countably infinite, discrete subsets of $X$ such that $C_{n_{k}} \subset \bar{U}_{n_{k}}, C_{n_{j}} \cap C_{n_{k}}=\varnothing$ if $j \neq k$, and $C_{n_{k}} \boxplus x_{0}$, where $1=n_{1}<n_{2}<\cdots$.

Let $Z=\cup_{k=1}^{\infty} C_{n_{k}} \cup\left\{x_{0}\right\}$. Then $Z$ is a closed subset of $X$ and is homeomorphic to the space $Y_{0}$.

K. Morita [9, Theorem 9.2] has shown that if $X \times Y$ is a Fréchet space (or equivalently, a hereditarily sequential space [3]), then $X$ is strongly Fréchet, or $Y$ is discrete.

As for sequential spaces, from Lemmas 2.2 and 2.3, we have

Proposition 2.4. Let $X$ be sequential, and $Y$ first countable. If $X \times Y$ is sequential, then $X$ satisfies condition (C), or $Y$ is locally countably compact.

\section{Proofs of Theorems 1.1 and 1.2, and some examples.}

Proof of Theorem 1.1. The necessity follows from Lemma 2.1(A) and Proposition 2.4 .

The sufficiency follows from [6, Proposition 4.D.4] and [13, Corollary 2.4].

Proof of Theorem 1.2. The sufficiency follows from [14, Corollary 4.4]. So we shall prove the necessity. 
For this purpose, let $X \times Y$ be symmetrizable. Then $X \times Y$ is sequential, for symmetrizable spaces are sequential [1].

Suppose $Y$ is not locally compact. Then $Y$ is not locally countably compact, for countably compact, semimetrizable spaces are compact [10, Corollary 2]. Thus $X$ satisfies condition (C) by Proposition 2.4. That $X$ is semimetrizable follows from Lemma 2.1(B).

Now, by the following Remark 3.1, we see that in the necessity of the condition of Theorem 1.1, the assumptions "each point of $X$ is a $G_{\delta}$-set" and " $Y$ is first countable" are essential.

The symbols $R, Q$, and $Z$ will denote, respectively, the reals, the rationals, and the integers, all with their usual topologies.

REMARK. 3.1. (A) Let $X$ be a compact, sequential space which is not Fréchet. In fact, such a space exists by [3, Example 7.1]. Then $X \times Q$ is sequential by [13, Corollary 2.4]. But $X$ is not strongly Fréchet, nor is $Q$ locally countably compact.

(B). Let $X$ be the quotient space $R / Z$ with $Z$ identified to a point. Then $X$ is a countable $\mathrm{CW}$-complex. Let $Y$ be the countable, symmetrizable space in [14, Example 3.2]. Then a subset $F$ of $Y$ is closed whenever $F \cap C_{i}$ is closed for every convergent sequence $C_{i}(i=0,1,2, \ldots)$ in $Y$, where $C_{0}=\{0\}$ $\cup\{1 / n ; n \in N\}, C_{i}=\{1 / i+1 / n ; n \in N\}$. Thus, in view of the proof of $[7$, Lemma 2.1], $X \times Y$ is sequential. But the Fréchet space $X$ is not strongly Fréchet, nor is $Y$ locally countably compact.

\section{The property "sequential space" in $X^{\omega}$.}

Proposition 4.1. Let $X^{\omega}$ be sequential. Then $X$ satisfies condition (C).

Proof. In case $X$ is countably compact, it is easy to check that a sequential space $X$ satisfies condition (C).

In case $X$ is not countably compact, the space $N$ may be regarded as a closed subset of $X$. Since $X \times N^{\omega}$ is a closed subset of $X^{\omega}, X \times N^{\omega}$ is sequential. Hence $X$ satisfies condition (C) by Proposition 2.4.

By Lemma 2.1(A) and Proposition 4.1, we have

Proposition 4.2. Let $X$ be Fréchet, or each point of $X$ be a $G_{\delta^{-s e t}}$. If $X^{\omega}$ is sequential, then $X$ is strongly Fréchet.

Lemma 4.3. Let $X$ have one of the properties (i), (ii) and (iii) in Theorem 1.3. If $X$ is strongly Fréchet, then $X$ is metrizable.

Proof. From [6, Theorem 9.11 and Corollary 9.10], we need only prove case (iii).

Let $\mathfrak{D}=\left\{e_{\alpha} ; \alpha \in A\right\}$ be the collection of cells in $X$. We shall prove the collection $\overline{\mathfrak{D}}=\left\{\bar{e}_{\alpha} ; \alpha \in A\right\}$ is point-finite.

Suppose that there is a point $x_{0} \in X$ such that infinitely many elements of $\bar{D}$ contain the point $x_{0}$. So we may assume each $\bar{e}_{\alpha_{n}}(n \in N)$ contains the point $x_{0}$. Let $F_{n}=\cup_{n=1}^{\infty} e_{\alpha_{n}}-\cup_{i=1}^{n} e_{\alpha_{i}}$. Then $x_{0} \in \bar{F}_{n}$ for $n \in N$. Since $X$ is strongly Fréchet, there exist $x_{n} \in F_{n}$ such that a sequence $\left\{x_{n}\right\}$ converges to some point $x^{\prime} \in X$. Let us put $K=\left\{x_{n} ; n \in N\right\} \cup\left\{x^{\prime}\right\}$. Then $K$ meets infinitely many elements of $\mathfrak{D}$. While, $K$ is a compact subset of a $\mathrm{CW}$-complex 
$X$. Thus $K$ meets only a finite number of elements of $\mathfrak{D}$. This is a contradiction. Hence the collection $\overline{\mathfrak{D}}$ is point-finite.

By a similar method, we can see that for each point $x$ of $X$, assuming only these $\bar{e}_{\alpha_{i}}(i=1,2, \ldots l)$ contain the point $x$, there is a neighborhood $U$ of $x$ such that $\bar{U} \subset \bar{e}_{\alpha_{1}} \cup \cdots \cup \bar{e}_{\alpha_{l}}$. Since $\bar{e}_{\alpha_{1}} \cup \cdots \cup \bar{e}_{\alpha_{l}}$ is metric, $U$ is metric. Hence $X$ is locally metrizable. Since a CW-complex is paracompact [9], $X$ is metrizable.

Proof of Theorem 1.3. From the hypothesis for $X$, each point of $X$ is a $G_{\delta^{-}}$ set. Thus, by Lemma 2.1(A), Proposition 4.1 and Lemma 4.3, $X$ is a metric space.

From the following example, we see that the product $X^{\omega}$ of a sequential (symmetrizable) space $X$ need not be sequential (symmetrizable) even if, for all $n \in N, X^{n}$ is sequential (symmetrizable).

EXAMPLE 4.4.Let $X$ be the symmetrizable space $Y$ in Remark 3.1(B). Then, in view of the proof of [7, Lemma 2.1], for all $n \in N, X^{n}$ is sequential and hence is symmetrizable by [14, Theorem 4.2], while $X$ is an $\aleph_{0}$-space but is not metrizable. Then, by Theorem $1.3, X^{\omega}$ is not even sequential and hence is not symmetrizable.

\section{REFERENCES}

1. A. V. Arhangel'skiĭ, Mappings and spaces, Uspehi Mat. Nauk 21 (1966), no. 4 (130), 133184 = Russian Math. Surveys 21 (1966), no. 4, 115-162. MR 37 \#3534.

2. S. P. Franklin, Spaces in which sequences suffice, Fund. Math. 57 (1965), 107-115. MR 31 \#5184.

3. - Spaces in which sequences suffice. II, Fund. Math. 61 (1967), 51-56. MR 36 \# 5882.

4. L. F. McAuley, A relation between perfect separability, completeness, and normality in semimetric spaces, Pacific J. Math. 6 (1956), 315-326. MR 18, 325.

5. E. A. Michael, $\kappa_{0}$-spaces, J. Math. Mech. 15 (1966), 983-1002. MR 34 \#6723.

6. - A quintuple quotient quest, General Topology and Appl. 2 (1972), 91-138. MR 46 \#8156.

7. J. Milnor, Construction of universal bundles. I, Ann. of Math. (2) 63 (1956), 272-284. MR $17,994$.

8. K. Morita, On spaces having the weak topology with respect to closed coverings, Proc. Japan Acad. 29 (1953), 537-543. MR 15, 977.

9. - Some results on $M$-spaces, Colloq. Math. Societatis János Bolyai 8, Topics in Topology, Keszthely, Hungary, 1972, pp. 489-503.

10. S. Nedev, Symmetrizable spaces and final compactness, Dokl. Akad. Nauk SSSR 175 (1967), 532-534 = Soviet Math. Dokl. 8 (1967), 890-892. MR 35 \# 7293.

11. R. C. Olson, Bi-quotient maps, countably bi-sequential spaces, and related topics, General Topology and Appl. 4 (1974), 1-lt28.

12. F. Siwiec, Sequence-covering and countably bi-quotient mappings, General Topology and Appl. 1 (1971), no. 2, 143-154. MR 44 \#5933.

13. Y. Tanaka, On quasi-k-spaces, Proc. Japan Acad. 46 (1970), 1074-1079. MR 45 \# 5946.

14. - On symmetric spaces, Proc. Japan Acad. 49 (1973), 106-111.

Department of Mathematics, Tokyo Gakugei University, 4-1-1 Nukuikita-machi, KogaNEI-SHI, TOKYO, JAPAN 